\title{
Study on Monitoring and Acquisition System of Stress throughout the Life Cycle of Vehicle
}

\author{
Shuang Shi ${ }^{1, a}$, Pengfei Feng ${ }^{1, b}$, Lingli $\operatorname{Han}^{1, c}$ \\ ${ }^{1}$ Anhui Sanlian University, Hefei, China \\ ashi1978117@163.com, b18063780@qq.com, c156689152@qq.com
}

\begin{abstract}
Keywords: stress collection; life cycle; reliability
Abstract. It is complex that the structure and operating environment of Vehicle. Using stress monitoring and acquisition system, we can collect temperature and humidity, load, vibration and other information in the whole life cycle of vehicle. The system architecture, hardware and software are designed to solve the problem that the data which is in need of reliability study. The practice has proved that the system has a good effect.
\end{abstract}

\section{Introduction}

Vehicle is a complex system, and it is made up of thousands of parts. The vehicle will be in varieties of environments throughout the life cycle. It will be subjected to various stresses, such as external environmental stress, internal functional stress and motion stress. The external environment stress and motion stress are more important than others.

The study of the reliability of vehicle is relied on reliability data collected. Bygone days, reliability data was collected at discrete stages of life cycle, so the large amount of data is discrete. At the same time, data has correlation and influence at different stages. The glitches in the whole life cycle is randomized, and they are related to facts such as environment, time, times, and operation methods. Because of the characteristics of reliability data, it is very difficult that collecting and tracking data. Traditional data collected is based on reports of test field, maintenance department and regular maintenance, so there are many problems like a little data, the glitches being not timely feedback, lack of basic data and so on. The data is not effective to analyze and study for reliability. With the development of internet of things, they can be realized that the overall perception of dynamic object, information transmission in real-time, and that analyzing and processing mass data in cloud computing and intelligent computing[1-3]. The technology of Internet of things can solve the problem of real-time data collection, transmission and mass data processing in the whole life cycle of vehicle, and maintenance plan can also be planned timely for enhancing vehicle reliability based on feedback information.

\section{System requirements}

According to the characteristics of vehicle, the monitoring and acquisition system of stress throughout life cycle needs the following characteristics

All data records can be collected safely and conveniently by the handheld acquisition device, base station of portable acquisition or fixed base station and so on. We can analyze and use the data in upper computer.

The system can monitor and gather environmental parameters, including temperature, humidity, acceleration, load, mileage and so on; The system with function of active electronic label can be controlled in reading distance and work mode. System internal clock is accurate and storage space is enough. 


\section{System design}

According to requirements, the system includes three parts: electronic vehicle recorder of stress, information acquisition device and information processing system in upper computer. The system framework is shown in Fig. 1

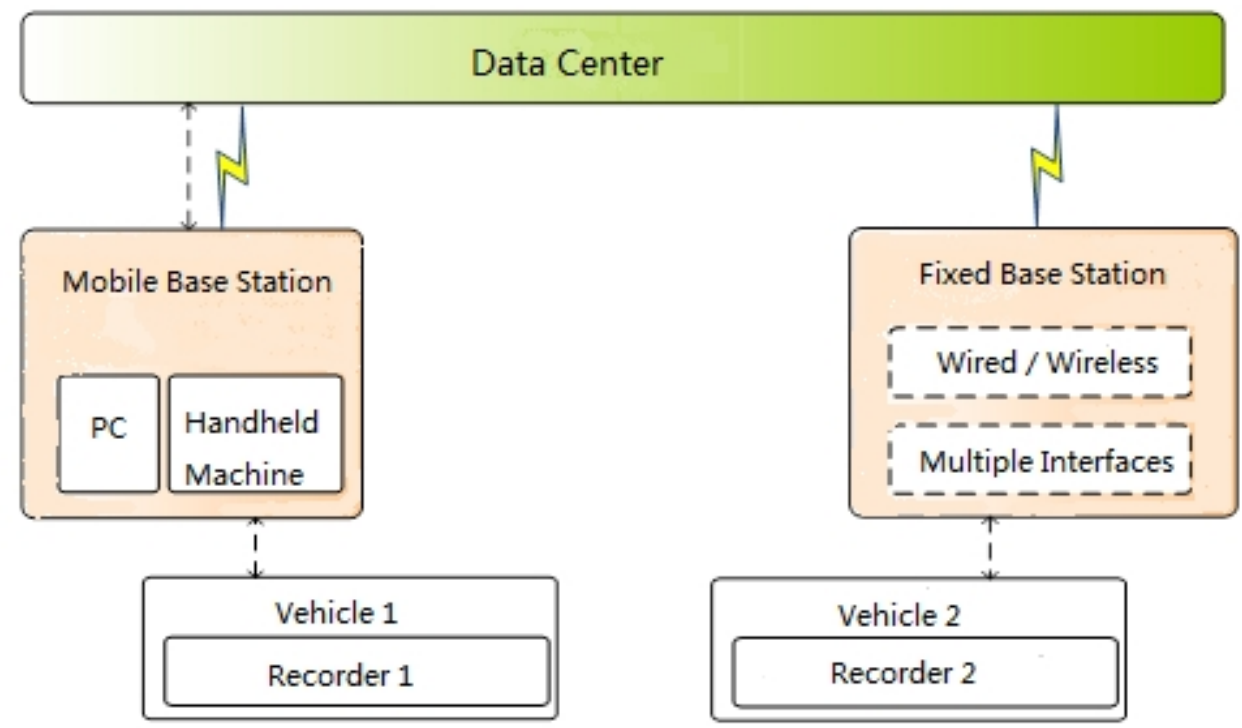

Fig. 1 system framework

\section{System composition}

In particular, the system consists of a recorder, a mobile transmission network and a data center. The recorder is installed and fixed on the vehicle and is responsible for collecting and recording the information of the vehicle's temperature, humidity, acceleration, load and mileage. The data center can input and output information to the recorder via the mobile transmission network, and complete the functions of reading the data or changing the working mode, the state and the correcting time. The data center stores all the stress information of the vehicle and can make statistics and analysis. The composition is shown in Fig. 2.
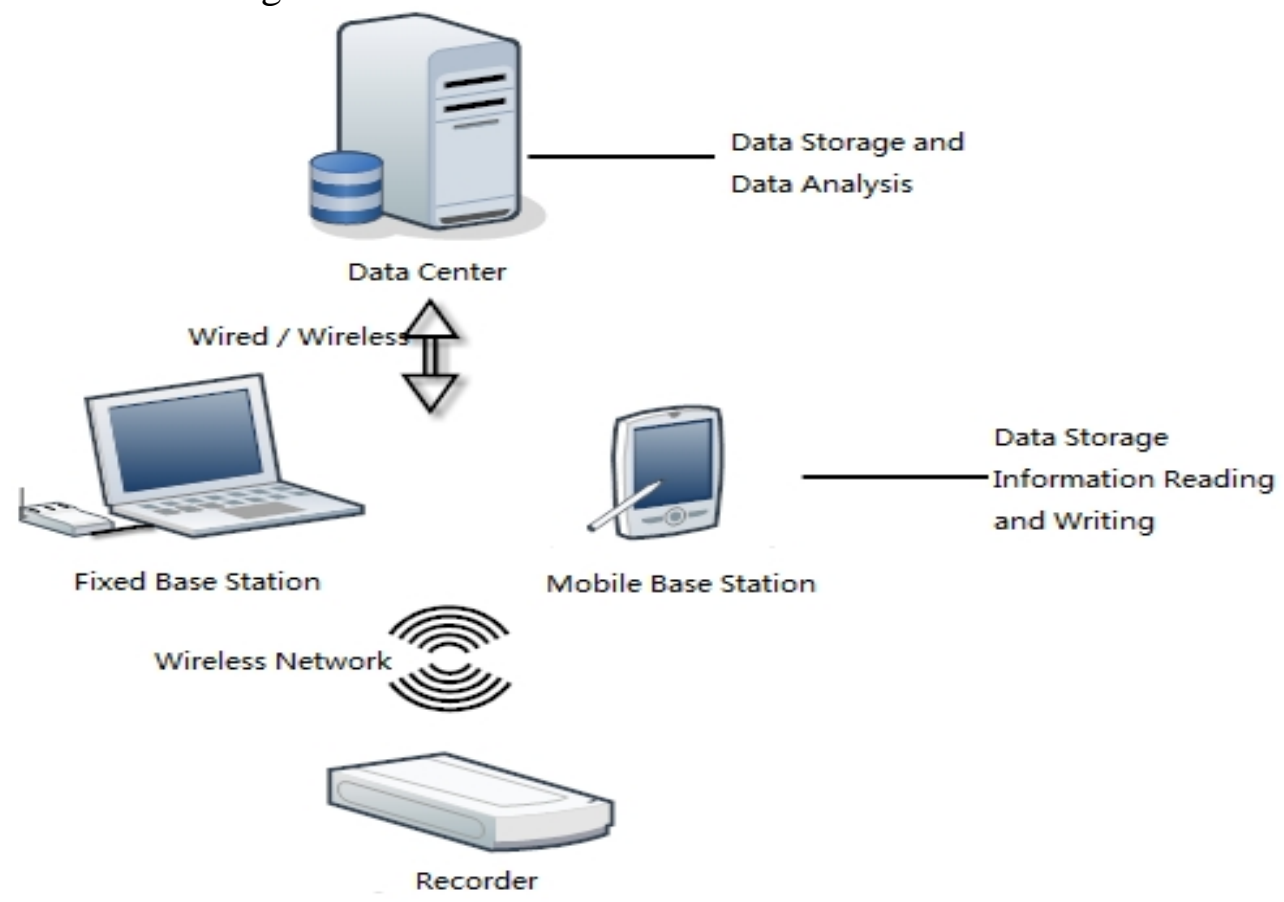

Fig. 2 equipment composition

\section{Hardware design}

The recorder with electronic label has unique ID coding. The parameters recorded are: time, temperature, humidity, acceleration, load, mileage, vibration and alarm information and so on. The 
recorder is installed on vehicle. Temperature and humidity sensors, weight sensors are installed at the bottom of vehicle. the acceleration sensor is installed in the internal of vehicle and three axis acceleration sensor that MEMS is used in the system. The MEMS is connected rigidly to ensure data accuracy. It can detect movement, displacement, vibration state. The body of recorder is installed in the driving room. High reliability memory is used in recorder to record stress information in whole the life cycle of vehicle. The design of recorder is shown in Fig. 3.

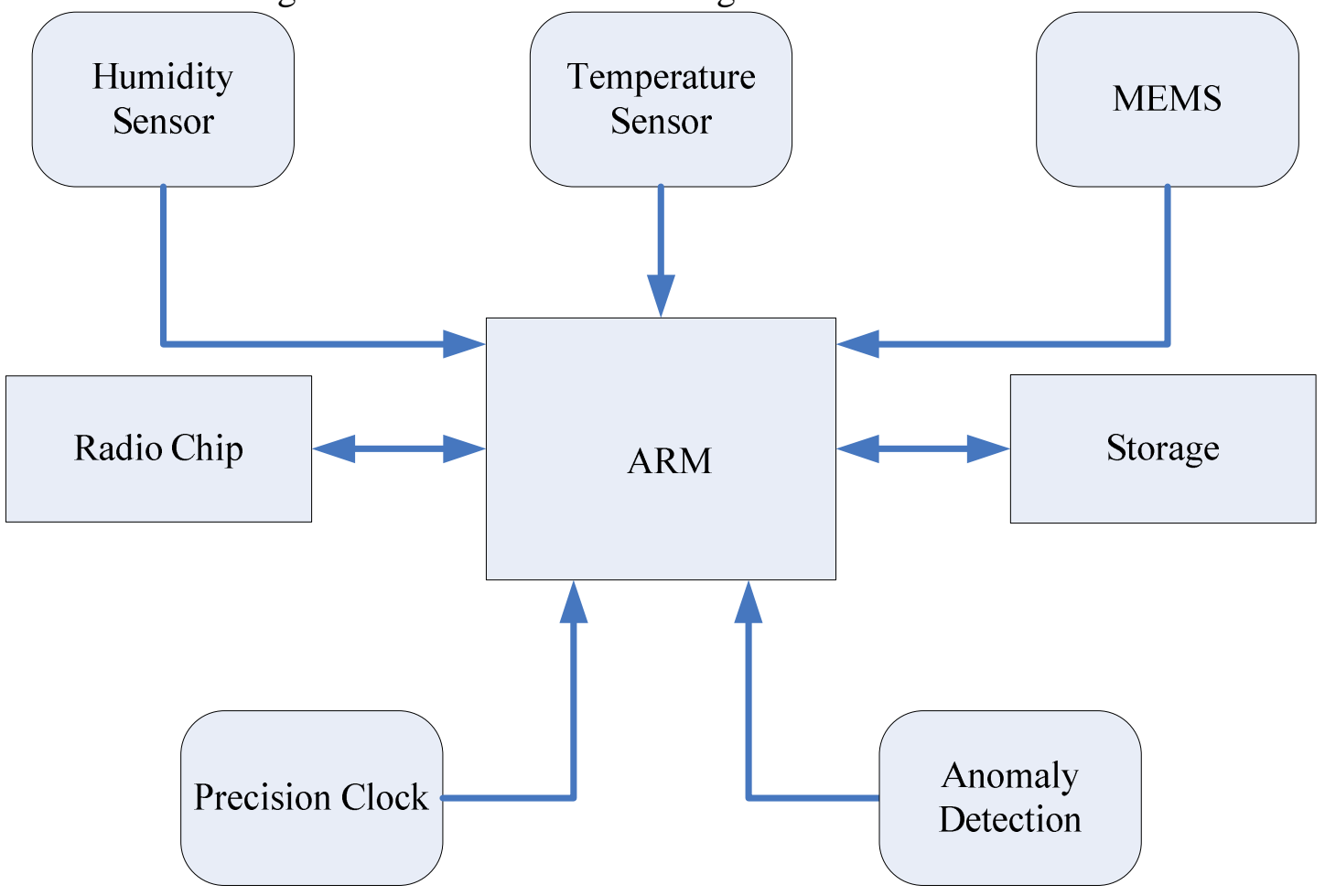

Fig. 3 design of recorder

(1) The processor uses 32 bit ARM with high performance and low power consumption, including multiple functional interfaces, which is convenient for expansion. Each recorder contains the only device number that can not be changed.

(2) The domestic Tang core chip is used to transfer radio data. Its physical layer is good security. Meanwhile, the transmission power can be adjusted by design of peripheral circuit to increase reliability for important information transmission.

(3) Storage circuit reserved for extensible location. When single chip capacity is insufficient, it can expand more than one chip. There is encryption mode in hardware design, and data is not easy to be cracked.

(4) MEMS acceleration sensor has many characteristics, such as three axis, high precision, ultra low power.

(5) A high precision real-time clock circuit is designed to ensure the stability of the recorder in long time without clock correction.

(6) The main circuit board and the sensor board are designed separately. They are installed on different parts of the vehicle. Low power consumption is designed for the circuit board, and large power components are designed in switch circuit for power consumption control. All circuit boards is designed highly reliable.

\section{Software design}

Function design of Software of data center

(1) Multi-function integrated design, including the reading and writing data, program upgrade instruction.

(2) Setting up the data rights and passwords for reading recorder.

(3) Supporting function of online upgrade (IAP).

(4) With the ability to initialize, configure, and manage the recorder. 
(5) With the ability to analyze and count data.

(6) With humanized interface.

\section{Function design of recorder}

(1) The embedded software in the recorder can upgrade via the wireless internet (OTA).

(2) The multi-function mode of recorder can be set, and the corresponding working mode is selected according to the occasion. The modes include silent acquisition mode, motion collection mode, interaction mode, storage mode.

(3) The recorder can be set the automatic switching mode, such as automatically switching to the motion acquisition mode as moving or automatically switching to the silent acquisition mode.

(4) All kinds of extreme value alarm can be set.

\section{Conclusions}

The reliability status and rule of reliability change are reflected by reliability data of vehicle in different life stages. Using the system studied in this paper, we can collect a lot of stress data, and provide effective samples for vehicle reliability assessment. Based on it the assessment results will be closer to the real state of vehicle.

\section{Acknowledgments}

This research is supported by the fund of education department of Anhui provincial (No. KJ2018A0599) and National Nature Science Foundation of China(No.51769027).

\section{References}

[1] http: / /www. autoidlabs. org/page. html [DB /OL] .

[2] International Telecommunication Union UIT. ITU Internet Reports2005: The Internet of Things[R], 2005.

[3] Qibo Sun, Jie Liu, ShanLi. Review of the concept architecture and key technology of the Internet of things[J]. Journal of Beijing University of Posts and Telecommunications, 2010, $33(3): 1-9$. 